\title{
Preservation planning and workflows for digital holdings at the Thomas Fisher Rare Book Library
}

\author{
Jess Whyte \\ University of Toronto Libraries \\ 130 St. George Street \\ Toronto, ON
}

\begin{abstract}
The goal of this practice-based work is to share experiences and findings with other digital preservation practitioners. The Thomas Fisher Rare Book Library Digital Preservation Pilot is a collaborative project involving the Thomas Fisher Rare Book Library, Information Technology Services at University of Toronto Libraries, and the TALint internship program at the school's Faculty of Information. Guidance was also provided by the Digital Curation Institute at the University of Toronto. The purpose of the project was to evaluate the extent of born-digital content at risk in the Fisher's collections, develop a workflow for accessioning, and establish a baseline level of preservation on the content. The following is an overview of that process, results, challenges, and recommendations for next steps.
\end{abstract}

\section{Keywords}

digital preservation, digital curation, manuscripts, rare books, workflow, bitcurator, fiwalk, md5deep, hfs2dfxml, bagger, forensic imaging

\section{INTRODUCTION}

The Thomas Fisher Rare Book Library is the largest repository of publicly accessible rare books and manuscripts in Canada. As part of the University of Toronto Libraries, the Fisher prioritizes the acquisition, preservation and accessibility of rare and special collections of local, national, and international significance. It serves both the University community and the general public. Until recently, born-digital accessions on legacy media formats at the Fisher were handled in the same manner as other artifacts - they were cataloged or included in the finding aid, placed in an archival box and put in a climate-controlled environment. The content on the disks was not examined or imaged.

As the University of Toronto Libraries (UTL) develops its digital preservation policies and workflows, these disks were identified by Steve Marks (the Digital Preservation Librarian) and myself (the UTL Digital Preservation intern) as an

Permission to make digital or hard copies of all or part of this work for personal or classroom use is granted without fee provided that copies are not made or distributed for profit or commercial advantage and that copies bear this notice and the full citation on the first page. To copy otherwise, to republish, to post on servers or to redistribute to lists, requires prior specific permission and/or a fee.

JCDL 2017 June 19-23, 2017, Toronto, ON, Canada

Copyright 2017 ACM X-XXXXX-XX-X/XX/XX ...\$15.00. ideal testing ground for some of these processes. The collection was: large enough to be significant; somewhat heterogeneous in terms of age, media and represented filesystems; comprised of content with clear boundaries (e.g. disks and drives, not relational databases or web archives); already selected and identified as significant; and, most importantly, at risk. Threats to the collection include: loss of content from media failure, hardware obsolescence or disaster; software obsolescence; declining digital donations; and insufficient representation data affecting the ability to render or locate items.

The scope for this project included all born-digital holdings at the TFRBL (published works and manuscripts). The scope did not include digitized collections created by the TFRBL, the creation of access copies, systematic individual file extraction and/or migration, or audio-visual materials (which are already addressed by ongoing media preservation work).

To date, over 1300 disk images across 115 manuscript collections or catalog items have been imaged and accompanying metadata created. The project is now moving into an analysis and reporting phase. This includes developing recommendations for future work in regards to access and the ability to provide scholars and researchers with materials in a manner that matches their research needs.

This work provides an overview of the project so far, the workflow developed (tools used, decision points, and development of in-house automation scripts), graphical representations of findings (failure rates, file types), and recommendations for future work.

\section{PROJECT TIMELINE}

The project took approximately 325 labour hours over an 18-month period. These hours included evaluating the extent of the content at risk, developing a workflow, piloting and tweaking that workflow, imaging the disks, and creating the metadata. This works out to approximately $15 \mathrm{~min} /$ object, but know that a straight-forward, FAT filesystem, 1.44MB diskette with no physical damage will take $5 \mathrm{~min}$ to move through the workflow, but a non-standard object requiring more hands-on intervention might take hours. Also, these time estimates do not include transferring objects to ITS's data protection platform or the ongoing management of those objects over the long term. The following is a narrative overview of that 18-month timeline divided into three phases.

\subsection{Phase 1}


Phase 1 of the project included creating an inventory of items, drafting a workflow, conducting time trials to establish feasibility and cost, and proposing the project to both Information Technology Services and the Thomas Fisher Rare Book Library. The elements of this collection-specific proposal were based on Becker et al's recommended elements of a preservation plan [2].

The inventory or audit of digital holdings at the Fisher involved querying both the catalog and finding aids with a set of terms related to digital objects (e.g. "disk", "disc","floppy", "usb", "laptop", "CD") and compiling those results. I often estimated the extent of digital content because some of the finding aids described, for example, "a box of disks" rather than "a box of 43 disks." Estimates were based on assuming items in the catalog represented a single disk or object, and, for the manuscripts, requesting a sample of six boxes and then making a rather ad-hoc estimate of 20 disks per mentioned box. Using this method, I estimated 3000 objects currently in the holdings and ended up with just over 1300 .

A workflow was then drafted based on research into how other institutions were structuring their processes for digital manuscripts. For this, I relied heavily on Martin Gengenbach's thesis The way we do it here: Mapping digital forensics workflows in collecting institutions [5], and Alice Prael and Amy Wickner's workflow example from the University of Maryland [7]. Once a workflow was drafted, time trials were conducted to estimate how long the project might take and its feasibility.

\subsection{Phase 2}

Phase 2 of the project included physically handling the disks, imaging them, creating metadata, generating checksums, bagging packages, submitting JIRA tickets to track bag locations, and, finally, writing those bags to tape on the IBM Tivoli Storage Manager system housed in ITS and an offsite location.

Equipment used in this phase include a Forensic Recovery Evidence Device (FRED) workstation and a Kryoflux floppy control card. For all 3.5" and 5.25" floppies, disk imaging was done using the Kryoflux software. For optical media, ZIP disks, portable hard drives, USB keys and other media, imaging was done using FTK Imager [1].

Metadata creation was automated through a shell script [8] which funneled images to either fiwalk [4] or hfs2dfxml [3] based on their filesystems. This work was done in the BitCurator environment [6], but, because the HFS filesystem accounted for $34 \%$ of the objects imaged in this project, the BitCurator GUI workflow wasn't well suited to the task (fiwalk, an underlying tool used in the BitCurator environment, does not currently support the HFS filesystem).

\subsection{Phase 3}

Phase 3 involves analysis of the collection, and the current work of reporting on the project and making recommendations for next steps.

\section{FINDINGS}

1308 objects were imaged with a failure rate of $17 \%(\sim 230$ objects were unreadable due to data corruption, mechanical failure of the disk, unavailability of hardware, or the use of now-unavailable proprietary backup software). A small amount of physical damage was discovered (e.g. missing sliders on disks) and noted, and no biological damage was
Table 1: Media Formats

\begin{tabular}{|c|c|}
\hline Medium & Amount \\
\hline 3.5" floppy & 925 \\
\hline Optical media & 217 \\
\hline $5.25 "$ floppy & 118 \\
\hline Syquest disk & 8 \\
\hline ZIP disk & 20 \\
\hline USB key & 9 \\
\hline Hard drive & 3 \\
\hline Other & 8 \\
\hline
\end{tabular}

found (based on a cursory visual inspection when I handled and processed each disk).

The items spanned a period of 33 years (1984 - 2017) with most being dated in the 1990s. Looking at Table 1: Media Formats, the low number of hard drives and USB keys, and the absence of any cloud drives or email records support this date range. This also might indicate (1) digital donations are dropping off, (2) donors are waiting to compile these items, and/or (3) donors are not aware the library is equipped to receive these items, or the digital donation process.

Common files types included jpegs, fonts and font metrics (the Fisher holds the archives for several presses), Corel/WP documents, and GIFs. Knowing common file types is a way of monitoring a collection, looking out for signs of possible software obsolescence issues or the rise of file formats that require a different approach to digital preservation (e.g. interactive applications). Fiwalk relies on a tool called libmagic to determine file type based on an identifier in the file signature known as the magic number. This was not always reliable. Files may not have a signature or magic number, or the signature may not be in the libmagic database. Unidentified file types accounted for $50 \%$ of the total.

\section{NEXT STEPS}

Recommendations for future work include: (1) communicating with potential donors about digital capabilities at the Fisher and asking about digital manuscripts, (2) Developing an access workflow (3) Exploring emulation tools like bwfla's EaaS, (4) Prioritizing items for the extraction and normalization of individual files, and (5) Consulting with researchers about their needs and preferences when working with this content.

\section{ACKNOWLEDGMENTS}

I would like to thank Steve Marks (Digital Preservation Librarian at UTL), Dr. Christoph Becker (Faculty of Information, University of Toronto), Natalya Rattan (Manuscripts Archivist at the Thomas Fisher Rare Book Library), and Jennifer Toews (Modern Manuscripts Librarian at the Thomas Fisher Rare Book Library). Funding for this project provided by University of Toronto Library's Toronto Academic Library Internship (TALint).

\section{REFERENCES}

[1] AccessData. Ftk imager lite http://accessdata.com/product-download/digitalforensics/ftk-imager-lite-version-3.1.1.

[2] C. Becker, H. Kulovits, M. Guttenbrunner, S. Strodl, A. Rauber, and H. Hofman. Systematic planning for 
digital preservation: Evaluating potential strategies and building preservation plans. International Journal on Digital Libraries, 10(4):133-157, 2009.

[3] D. Dietrich. hfs2dfxml https://github.com/cul-it/hfs2dfxml, 2015.

[4] S. Garfinkel. Automating disk forensic processing with sleuthkit, xml and python. Systematic Approaches to Digital Forensic Engineering, 2009. SADFE'09, IEEE, May 2009.

[5] M. Gengenbach. The way we do it here: Mapping digital forensics workflows in collecting institutions. The University of North Carolina at Chapel Hill, Chapel Hill, North Carolina, 2012.

[6] C. Lee, M. Kirschenbaum, P. Olsen, A. Chassanoff, and K. Woods. Bitcurator: tools and techniques for digital forensics in collecting institutions. D-Lib Magazine, 18(5/6):14-21, 2012.

[7] A. Prael and A. Wickner. Getting to know fred: Introducing workflows for born-digital content. Library Research and Innovative Practice Forum, June 2015.

[8] J. Whyte. Disk-id-md5deep https://github.com/jesswhyte/disk-id-md5deep, 2016. 